\title{
Quality of Life of Haemodialysis Patients and Complications of CVC and AA
}

\author{
Despo Theodorou ${ }^{1, *}$, Anna Papasavva ${ }^{1}$, and Antonia Kalli ${ }^{2}$ \\ ${ }^{1}$ Department of Administrating Health Units, Open University of Cyprus, Nicosia, Cyprus \\ ${ }^{2}$ Department of Health Sciences, School of Science, European University of Cyprus, Nicosia, Cyprus
}

*Corresponding author: Despo Theodorou, Adult Educator Trainer, Department of Administrating Health Units, Open University of Cyprus, Ioanni Notara 9, 6058, Larnaca, Cyprus, Tel: 00357-99559908; E-mail: despotheo@yahoo.gr

Received: 11 Sep, 2021 | Accepted: 28 Sep, 2021 | Published: 09 Oct, 2021

Citation: Theodorou D, Papasavva A, Kalli A (2021) Quality of Life of Haemodialysis Patients and Complications of CVC and AA. Int J Nephrol Kidney Fail 7(4): dx.doi.org/10.16966/2380-5498.219

Copyright: (C) 2021 Theodorou D, et al. This is an open-access article distributed under the terms of the Creative Commons Attribution License, which permits unrestricted use, distribution, and reproduction in any medium, provided the original author and source are credited.

\begin{abstract}
Background: The increase in life expectancy, as well as in chronic diseases, has resulted in giving emphasis not only to medical interventions, but to the continuous care of the patient. The measurement of Quality of Life (QOL) of haemodialysis patients provides information relevant to their needs and the consequences of haemodialysis in their life, which could be used to implement interventions that will lead to the improvement of their QOL.

Aim: The aim of this research was to imprint the QOL of haemodialysis patients treated at Larnaca and Nicosia General Hospital (GH) and their correlation with demographic characteristics and health information, as well as to record and compare the complications of Central Venus Catheter (CVC) and Arteriovenous Anastomosis (AA).

Methods: The sample of this research consisted of 193 haemodialysis patients who attended the haemodialysis units of Larnaca and Nicosia GH. During the period between 26/2/2014 and 15/4/2014, the collection of data was made possible with the use of questionnaire of demographic characteristics and patient health data, questionnaire of WHOQOL-BREF- Greek version and questionnaire of complications of vascular access haemodialysis patients. For the statistical analysis and processing of data the software SPSS 20 was used.

Results: The QOL of most haemodialysis patients is located at a medium level (men $54,26 \pm 14,74$, women $46,83 \pm 13,09$ ) and is lower in comparison to the healthy population $(P<0,00$, DM of physical health $=-42,31$, mental health $=-14,20$, social relations $=-11,88)$. However, they present lower scores in the factor of physical health $(32,27 \pm 19,10)$ in relation to the other factors (mental health $52,59 \pm 21,21$, social relations $59,61 \pm 15,75$, environment $68,15 \pm 12,85)$. Additionally, female haemodialysis patients present lower $Q O L$ scores in relation to the males $(P=0,003, B=-6,53)$, the educational level affects positively the QOL $(P<0,001, B=3,18)$, whereas the accompanying health problems $(P=0,001, B=-2,95)$ and the number of complications in vascular access $(P=0,001, B=-2,93)$ affect it negatively. Furthermore, the QOL of haemodialysis patients with $A A$ appears better in the factor of mental health in relation to haemodialysis patients with CVC $(P=0,008$, Difference Mean $(D M)=2,06)$. Regarding the complications of vascular access, the patients with CVC present higher percentages in local infections $(P<0,001$, Percentage Difference $(P D)=27,37)$, bacteremia $(P$ $<0,001, P D=39,94)$, low blood supply $(P<0,001, P D=38,06)$, increased venous pressure $(P=0,001, P D=9,72)$ and narrowing or occlusion of the central veins $(P=0,024, P D=10,85)$ in relation to the patients with $A A$, whereas patients with $A A$ present aneurysms in relatively high percentages $(24,32 \%)$.

Conclusions: The QOL of haemodialysis patients is at a medium level and below the healthy population. Providing holistic health care could contribute to an improvement in their QOL. Patients with AA present less complications of vascular access and higher percentages of mental health in relation to the patients with CVC, which is why AA is recommended as first choice of permanent vascular access.
\end{abstract}

Keywords: Quality of life; Chronic renal failure; Haemodialysis; Central venous catheter; Arteriovenous anastomosis

\section{Introduction}

In recent decades, the improvement of human living conditions and the development of medical science and technology have led to an increase in life expectancy and chronic diseases, resulting in an emphasis not only on medical interventions and treatments but also on continuous monitoring and health care [1].

End-stage renal disease is a chronic disease that causes a high level of disability in various areas of patients' lives and leads to reduced QOL. The availability of various renal function replacement therapies has reduced the severity of the symptoms and led to increased patient survival. But long-term dialysis treatment often leads to loss of freedom, dependence on caregivers, disturbances in family and social life, and a reduction or loss of income. Because of these reasons, all aspects of the patient's life-physical, psychological, socioeconomic, and environmental are adversely affected and lead to reduced QOL. Therefore, improving QOL is a challenge for health care providers [2].

Vascular access for dialysis is a vascular surgery that includes the CVC, AA and Arteriovenous Graft (AG). They are the cornerstone of dialysis, but with their complications they are a source of increased 
morbidity, hospital care and costs and contribute to the reduction of QOL of haemodialysis patients [3].

In the Unites States of American, Chronic Renal Failure (CRF) is more common among adults aged 50 to 70 years. Adults with diabetes or hypertension, or both, have a higher risk of developing CRF than those without the disease. More specifically, about 1 in 3 adults with diabetes and 1 in 5 adults with hypertension have CRF. In Canada, 1 in 10 Canadians has kidney disease, and even more millions are at risk. In addition, the number of Canadians being treated for kidney failure has more than tripled in 20 years. 53\% of young patients with renal insufficiency are 65 years of age or older. Regarding the main causes of kidney failure, diabetes (35\%) and hypertension (16\%) are also here. Dialysis is the most common treatment for kidney failure and costs the healthcare system an average of $\$ 83,000$ per patient per year [4].

The purpose of the present study is to record the QOL of haemodialysis patients of General Hospital in Larnaca and Nicosia as well as to record and compare the complications of CVC and AA. It includes the General part which refers to QOL, chronic renal failure, dialysis, vascular access, and its complications, as well as a literature review of studies related to QOL and vascular access of dialysis patients. The Special Part follows, which presents the methodology of the research work and its results, discusses, and comments on the results, and finally records the conclusions that emerge and the suggestions that are proposed.

\section{Methodology}

\section{Design}

The data collection was carried out using three questionnaires (The World Health Organization/Quality of Life-Bref (WHOQOL-BREF), Questionnaire of complications of vascular access of haemodialysis patients) which were given to the dialysis patients upon their arrival at the extrarenal dialysis units of the General Hospital of Larnaca and Nicosia [5]. Along with the three questionnaires, the dialysis patients were given the consent form which included the purpose of the investigation, the voluntary and anonymous participation of the patient, his written consent for participation, the procedure for conducting the investigation and the procedure for submitting complaints or grievances by of the participants.

\section{Sample and data collection}

The dialysis patients who came to the extrarenal dialysis units of Larnaca and Nicosia during the period between 26/2/2014 and $15 / 4 / 2014$ constituted the population from which the sample of the present quantitative research came. The criteria set for the participation of dialysis patients in the research were the possibility of communication in the Greek language, the satisfactory level of cooperation and perceptual ability and to be permanent residents of Cyprus. The number of patients who were registered in the two hospitals and followed the dialysis treatment program during the study period was 256 . However, the participation criteria were met by 242 patients, of whom 193 accepted to participate in the study, while others for personal or other reasons refused. More specifically, 66 dialysis patients were registered in the General Hospital of Larnaca, of which 2 did not meet the criteria and 2 refused, with the result that the participation reached 96.9\%, while in the General Hospital of Nicosia, 190 were registered, by of which 12 did not meet the criteria and 47 refused, with the result that the participation reached $73.6 \%$. In total, the participation from both hospitals reached $85.25 \%$.

The data collection of the present research was done after the permission for conducting the research was previously obtained from the Commissioner for Personal Data Protection, the Cyprus Bioethics Committee and the Ministry of Health. In addition, permission was obtained from the Directors of the General Hospital of Larnaca and Nicosia, as well as from the Directors of the dialysis units of the two hospitals. When the dialysis patients arrived at the dialysis unit, the two researchers were informed of the purpose of the investigation and were given all the information regarding the anonymity and security of their personal data from the consent form. After studying quietly and in their own time, if necessary, the questionnaires and giving them answers to all their questions, they decided whether they wanted to participate in the research. Then, after giving their signed consent, the questionnaires were completed either by the patients themselves or by the researchers after an interview and access to the medical records of the participants. Finally, the forms were sealed in separate envelopes and placed in a special secured box located in the cleaning units. Each patient was given a unique code which was written on the questionnaires. When the data collection was complete, the data was transferred to an excel file which contained only the participant's password. The database did not contain the patient's name so that personal data could be secured. All information in printed or electronic form upon completion of the investigation will be deleted and destroyed.

\section{Statistical analysis}

SPSS 20 software was used for the statistical analysis and processing of the research data, which is a data management and analysis program that covers all the techniques of tabulation and data analysis. The elaboration resulted in the basic statistical measurements, distributions, mean values, and control of the degree of significance for the hypotheses that require group comparisons.

\section{Results}

The total number of dialysis patients participating in the study was 193 with a Means age of 69.55 years and a Standard Deviation (SD) of $12.43,128$ of them were men $(66.32 \%)$ with an average age of 69.43 years and so on. 12.36 and the 65 women (33.68\%) with an average age of 69.78 years and a SD of 12.67 .116 of them $(60.10 \%)$ stated that they live in an urban area, 74 (38.34\%) in a rural area, while $3(1.55 \%)$ did not complete the specific question.

\section{Sample health problems}

Most of the participants in the research, in addition to the problem of ESCRF (End Stage Chronic Renal Failure) and haemodialysis, also had health problems that afflicted them and had a negative impact on their health and QOL, namely (Figure 1):

a) 99 suffered from hypertension,

b) 62 suffered from diabetes,

c) 59 had heart problems.

For 127 of them (65.80\% of the sample) ESCRF was the problem with the most serious effects on their lives (restriction, fatigue, discomfort, dizziness, pain), for $8(4,15 \%)$ all the problems, for the $7(3,63 \%)$ the problems in the lower extremities, for the $6(3,11 \%)$ the heart problems, for the $3(1,55 \%)$ the problem with the vision, for the 2 $(1,04 \%)$ diabetes, for $2(1.04 \%)$ chronic mental problems, for $2(1,04 \%)$ the problem with the spine, for $1(0,52 \%)$ hypertension, for $1(0,52 \%)$ arthritis or rheumatism, for $1(0,52 \%)$ cataracts, for $1(0,52 \%)$ urethral stricture, for $1(0,52 \%)$ burns , and for $1(0,52 \%)$ none of his problems. 30 of them $(15,54 \%)$ did not answer this question. It is obvious that in most dialysis participants, dialysis was the problem that had the most serious impact on their lives because it caused them discomfort and limited their various activities. 


\section{ACCOMPANYING PROBLEMS}

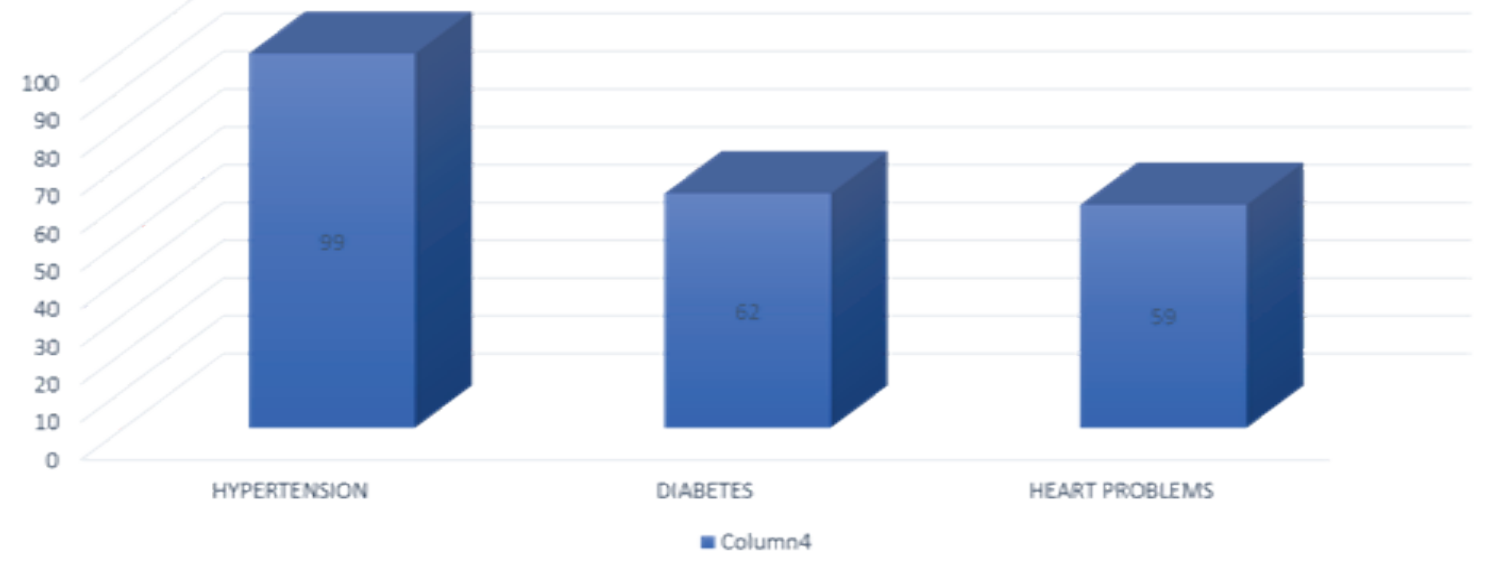

Figure 1: Sample health problems.

\section{CRF-related health data of the sample}

According to the participants and the data contained in their medical records, the primary cause of CRF in most of the sample was hypertension and diabetes mellitus. These are followed by medication, polycystic ovary disease and kidney stones. Specifically, the primary cause of CRF in 41 participants $(21,24 \%)$ was hypertension, in 34 (17.62\%) hypertension and diabetes mellitus, in $27(13,99 \%)$ diabetes mellitus, in $19(9,84 \%)$ medication, in 19 (9.84\%) of unknown etiology, in $8(4,15 \%)$ polycystic ovary disease, in $5(2,59 \%)$ kidney stones, in $5(2.59) \%)$ hereditary causes, in $4(2,07 \%)$ hypertension and other health problems, in $3(1,55 \%)$ hypertension and medication, in 2 $(1,04 \%)$ hypertension and polycystic ovary disease, in $2(1,04 \%)$ glomerulonephritis, in $2(1,04 \%)$ the diabetes mellitus and medication, in $1(0,52 \%)$ hypertension and glomerulonephritis, in $1(0,52 \%)$ hypertension and kidney stones, in $1(0,52 \%)$ diabetes mellitus and kidney stones and $19(9,84 \%)$ various other causes.

Regarding the time that the participants were in a haemodialysis program, the results showed that 60 patients $(31.09 \%)$ were less than or equal to one year, while $130(67.36 \%)$ were more than a year. Three patients (1.55\%) did not provide data on this question. Regarding the type of vascular access of the sample, 111 participants $(57.51 \%)$ had AA, 72 (37.31\%) CVC and 10 (5.18\%) AG. That is, most patients had AA.

Figure 2 below shows the distribution of the type of vascular access by province. The patients who were included in the dialysis program and participated in the study were $62(32.12 \%)$ from the province of Larnaca and $131(67,88 \%)$ from the province of Nicosia. 36 of the Larnaca sample (58.1\%) had CVC vascular access, 25 (40,3\%) AA and $1(1.6 \%) \mathrm{AG}$, while 86 participants from the province of Nicosia (65.6\%) had AA, 36 (27,5\%) CVC and 9 (6.9\%) AG. It is obvious that in the province of Larnaca the CVC prevails in contrast to the province of Nicosia which is dominated by the AA.

\section{Quality of Life of the sample}

The QOL of most dialysis participants is moderate. It was also found that the physical health factor has a much lower score than the other factors, while the environmental factor has the highest scores.
In addition, comparing the QOL of the two sexes it is obvious that women have lower QOL ( $\mathrm{p}=0,003$ ). It is not possible to compare with the healthy population of Cyprus because the research sample consists only of haemodialysis patients (Table 1).

Comparing in table 2 the averages of the QOL factors of the sample with those of the healthy working population "Psychometric properties of WHOQOL-BREF in groups of Greek patients and healthy individuals: Cultural adaptation with the integration of new questions" by Tzinieri-Kokkosi (2012), found that there is a statistically significant difference in all factors with $\mathrm{p}<0.001$. Specifically, the QOL of haemodialysis in the factors of physical health, mental health and social relationships is lower than in the healthy population. Regarding the environmental factor in which the research sample has a higher average compared to the healthy population, it is not possible to compare because the healthy population with which it is compared was in a different environment. Table 2 is with complete statistics.

\section{Type of vascular access and Quality of Life}

Table 3 presents the averages of QOL per category of vascular access (AA, CVC and AG). The values of the CVC factors used were the initial (row scores). There were only 10 patients in the sample with AG, so no comparative statistical test was used for this group. Moreover, it was not part of the original purpose of the investigation.

The average QOL in the physical health factor in patients with AA is 21.25 while in patients with CVC it is 19.76 and in patients with AG 19.70. In the mental health factor, patients with AA and AG show better results with 19.46 and 18.10 respectively compared to patients with CVC who have 17.40. In the factor social relations and environment all 3 groups range in the same statistical results. In total, the average number of dialysis patients with $\mathrm{AA}$ is 87.84 , with CVC, 83.47 and with AG 83.20.

\section{Comparison of vascular access of complications in patient with AA and CVC}

Graphs $1 \& 2$ show the number of vascular access complications in patients with AA and CVC respectively. It is observed that $48 \%$ of patients with AA had no complications related to their vascular 


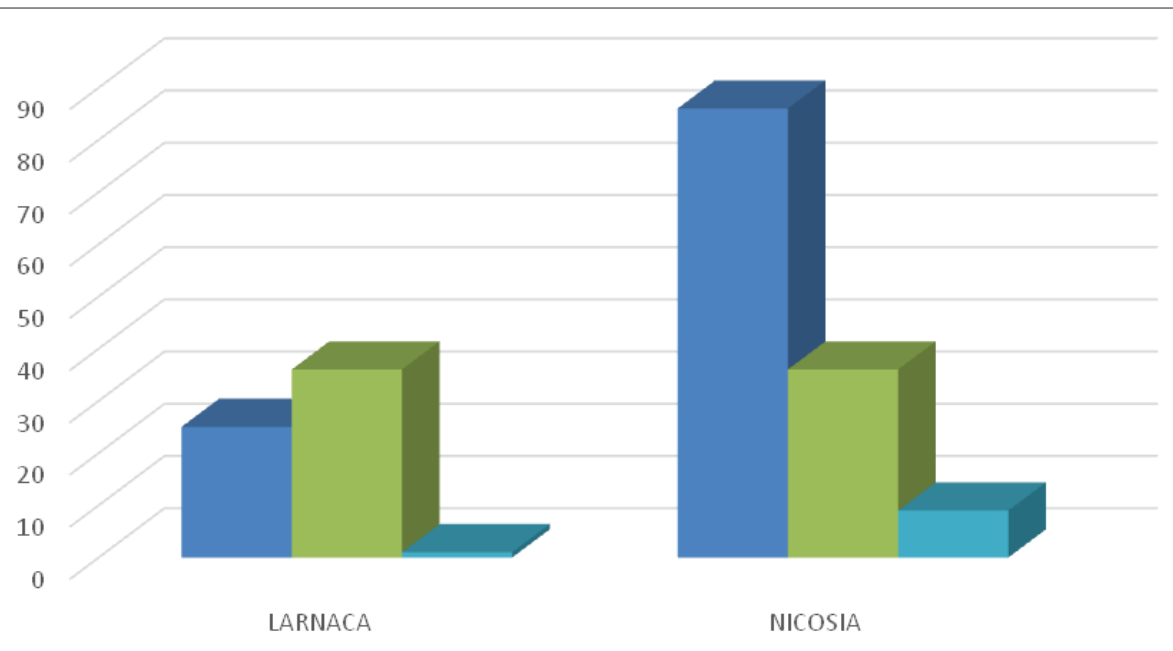

$\square \mathrm{AA} \backsim \mathrm{CVC} \square \mathrm{AG}$

Figure 2: Distribution of the type of vascular access by province.

Table 1: Participants' quality of life scores ( $N=193)$.

\begin{tabular}{|l|c|c|c|c|}
\hline \multirow{2}{*}{\multicolumn{1}{c|}{ Quality of life }} & \multicolumn{2}{c|}{ Male (N=128) } & \multicolumn{2}{c|}{ Female (N=65) } \\
\cline { 2 - 5 } & $\mathrm{M}$ & SD & M & SD \\
\hline Physical health & 35,28 & 20,06 & 26,36 & 15,58 \\
\hline Mental health & 56,08 & 20,96 & 45,71 & 20,17 \\
\hline Social relationships & 60,70 & 15,60 & 57,45 & 15,95 \\
\hline Environment & 70,25 & 12,09 & 64,03 & 13,38 \\
\hline Overall score QOL & 54,26 & 14,74 & 46,83 & 13,09 \\
\hline QOL general & 3,27 & 0,85 & 3,03 & 0,73 \\
\hline General health satisfaction & 2,91 & 1,14 & 2,57 & 1,12 \\
\hline
\end{tabular}

M: Average; SD: Standard Deviation

Table 2: Comparison of mean QOL factors of the sample with those of the healthy working population.

\begin{tabular}{|l|c|c|l|l|l|}
\hline $\begin{array}{c}\text { Quality of } \\
\text { life }\end{array}$ & $\begin{array}{c}\text { Sample } \\
\mathbf{( N = 1 9 3 )} \\
\mathbf{M}(\mathbf{S D})\end{array}$ & $\begin{array}{c}\text { Healthy } \\
\mathbf{( N = 6 7 )} \\
\mathbf{M}(\mathbf{S D})\end{array}$ & P-value & $\begin{array}{c}\text { Mean } \\
\text { (M) }\end{array}$ & $\begin{array}{c}\mathbf{9 5 \%} \\
\text { Confidence } \\
\text { interval }\end{array}$ \\
\hline $\begin{array}{l}\text { Physical } \\
\text { health }\end{array}$ & $\begin{array}{c}32,27 \\
(19,10)\end{array}$ & $\begin{array}{c}74,58 \\
(13,40)\end{array}$ & $<0,001$ & $-42,31$ & $(-47,28,-37,34)$ \\
\hline $\begin{array}{l}\text { Mental } \\
\text { health }\end{array}$ & $\begin{array}{c}52,59 \\
(21,21)\end{array}$ & $\begin{array}{c}66,79 \\
(12,95)\end{array}$ & $<0,001$ & $-14,20$ & $(-19,62,-8,78)$ \\
\hline $\begin{array}{l}\text { Social } \\
\text { relationships }\end{array}$ & $\begin{array}{c}59,61 \\
(15,75)\end{array}$ & $\begin{array}{c}71,49 \\
(13,70)\end{array}$ & $<0,001$ & $-11,88$ & $(-16,14,-7,62)$ \\
\hline Environment & $\begin{array}{c}68,15 \\
(12,85)\end{array}$ & $\begin{array}{c}54,06 \\
(11,69)\end{array}$ & $<0,001$ & 14,09 & $(10,58,17,60)$ \\
\hline
\end{tabular}

M: Average; SD: Standard Deviation

The scores of the QOL factors range from 0-100. Higher scores indicate better QOL. Statistically significant difference with $\mathrm{p}<0.001$.

access, $42 \%$ had 1 complication, $7 \%$ had 2 complications, $2 \%$ had 3 complications and $1 \%$ had 4 complications. Regarding patients with CVC, $22 \%$ of them had no complications, $32 \%$ had 1 complication, $27 \%$ had 2 complications, $7 \%$ had 3 complications, $7 \%$ had 4 complications, $4 \%$ had 5 complications and $1 \%$ had 6 complications. The percentage of patients who did not have complications in their vascular access was higher in patients with AA.
Table 3: Mean and standard deviations of QOL factors per type of vascular access ( $\mathrm{N}=193$ ).

\begin{tabular}{|l|c|c|c|}
\hline Quality of life & $\begin{array}{c}\text { Arteriovenous } \\
\text { Anastomosis } \\
\text { AA (N=111) }\end{array}$ & $\begin{array}{c}\text { Central Venous } \\
\text { Catheter CVC } \\
\mathbf{( N = 7 2 )}\end{array}$ & $\begin{array}{c}\text { Arteriovenous } \\
\text { Graft } \\
\text { AG (N=10) }\end{array}$ \\
\hline Physical health & $21,25(7,06)$ & $19,76(6,52)$ & $19,70(7,26)$ \\
\hline Mental health & $19,46(5,08)$ & $17,40(4,98)$ & $18,10(4,56)$ \\
\hline $\begin{array}{l}\text { Social } \\
\text { relationships }\end{array}$ & $17,07(3,32)$ & $16,76(2,85)$ & $16,40(3,50)$ \\
\hline Environment & $30,05(3,97)$ & $29,54(4,24)$ & $29,00(4,92)$ \\
\hline Total score & $87,84(16,57)$ & $83,47(15,84)$ & $83,20(16,43)$ \\
\hline
\end{tabular}

AA: Arteriovenous Anastomosis; CVC: Central Venous Catheter; AG: Arteriovenous Graft

Note: The scores on physical health range from 9-45, mental health from 6-30, social relationships from 5-25, environment from 8-40 and the overall QOL score from 28-140. Higher scores indicate better QOL.

\section{Treatment of Vascular access complications in patients with $\mathrm{AA}$ and $\mathrm{CVC}$}

Table 4 shows the percentage comparison of the various complications of vascular access in patients with AA and CVC. Problems related to vascular access occur in both patients with AA and patients with CVC, however, as shown in the figures above, more complications occur in patients with CVC. Specifically, 21 patients presented with local infection, which corresponds to $29.17 \%$ of the total population of patients with CVC, compared to 2 patients with $\mathrm{AA}$, which correspond to $1.80 \%$ of the total population of patients with AA. The result of this comparison is statistically very significant with a P-value $<0.001$, a percentage difference of 17.08 and a $95 \%$ confidence difference period ranging from 17.08 to 38.78 . There was also a statistically significant difference in bacteremia with a P-value $<0.001$, a percentage difference of 39.94 and a $95 \%$ confidence interval of the difference ranging from 27.68 to 51.69 . Specifically, 32 patients with CVC (44.44\%) had bacteremia compared to 5 patients with AA (4.50\%).

Regarding the dysfunction in the vascular access, 30 arterial patients showed low arterial flow (41.67\%) compared to 4 patients 


\section{Patients with Arteriovenous Anastomosis}

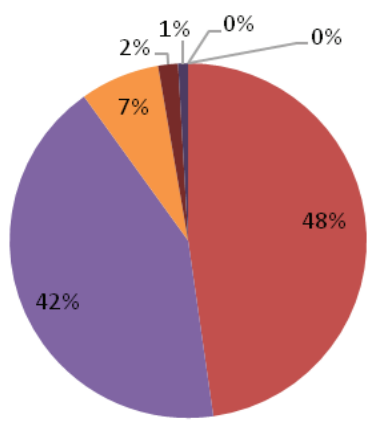

0 complications

1 complication

2 complications

- 3 complications

- 4 complications

- 5 complications

6 complications

Graph 1: Distribution of number of vascular access complications in patients with Arteriovenous Anastomosis (AA) ( $N=111)$.

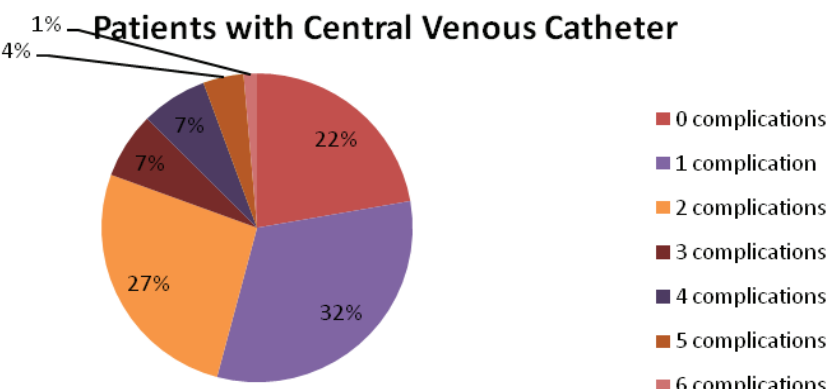

Graph 2: Distribution of number of vascular access complications in patients with Central Venous Catheter (CVC) $(\mathrm{N}=72)$.

with AA (3.60\%). The difference is statistically significant with P-value $<0.001$, percentage difference of the comparison 38.06 and the $95 \%$ confidence interval of the difference ranges from 26.14 to 49.80 . In addition, 7 patients with CVC (9.72\%) experienced increased venous pressure during dialysis, while patients with AA did not experience this complication. In this case, too, the difference is statistically significant with $\mathrm{P}$-value $=0.001$, percentage difference of the comparison 9.72 and the $95 \%$ confidence interval of the difference ranging from 3.76 to 18.74. Regarding the narrowing or obstruction of central veins, the difference is again statistically significant with $\mathrm{P}$-value $=0.024$, percentage difference 10.85 and the $95 \%$ confidence interval of the difference ranging from 1.24 to 21.85 . Specifically, 13 patients with CVC (18.06\%) had this complication compared to 8 patients with AA (7.21\%).

Finally, aneurysms occurred in 27 patients with AA (24.32\%), while patients with CVC did not present this complication. The difference is statistically significant here too with a $\mathrm{P}$-value $<0.001$, a percentage difference of 24.32 and the $95 \%$ confidence interval of the difference ranging from 15.66 to 33.08. As a result, patients with CVC had a higher percentage of local infections, bacteremia's, low blood flow, increased venous pressure, and central venous stenosis or obstruction compared with patients with AA, while patients with AA had a relatively high percentage of aneurysms $(24.32 \%)$.

\section{Discussion}

The results showed that the participants in the study, 193 dialysis in total, had an average age of 69.55 years and a standard deviation of
12.43. Based on data from the UK Renal Registry $14^{\text {th }}$ annual report [6] which states that the average age of patients undergoing haemodialysis in 2010 in the United Kingdom was 66.3 years, as with According to data from various studies such as the study of Panagopoulou A, according to which the average age of dialysis patients was 57.6, it is found that haemodialysis patients who participated in the study have a higher life expectancy [7].

In addition, 128 of the participants were men (66.32\%) and 65 women $(33.68 \%)$, which shows that the percentage of haemodialysis men is almost twice the percentage of haemodialysis women and agrees with the data reported. in the United Kingdom (UK) Renal Registry $14^{\text {th }}$ annual report on the prevalence of dialysis men in the UK, which was almost twice as high as women [6], as well as the Pelayo RA, et al. study in which $66 \%$ of the participants were men and $34 \%$ women [8]. $60.10 \%$ of the dialysis patients in the sample stated that they live in an urban area, therefore they are more likely to have fewer problems with going to the treatment plants, due to the short distance and the existence of means of transport. However, $38.34 \%$ who live in a rural area face more difficulties, especially those who are left alone and cannot drive.

According to the Kidney Foundation of Canada transportation to and from dialysis can be a significant and costly challenge [6]. In her research, Grilla E reports that 34 dialysis patients living in rural and mountainous areas were forced to change their place of residence to be close to the artificial kidney unit [9]. Regarding the educational level of the participants, access to primary education was $55.44 \%$, to secondary education $33.68 \%$ and to tertiary education $10.88 \%$. It is obvious that the majority had access to primary education, and this is justified by the fact that most of them are elderly and therefore born in the 40's and 50's, where very few could go to high school due to economic conditions and culture. Regarding their marital status, $76.17 \%$ of the participants were married, $9.33 \%$ widowed, $7.25 \%$ unmarried and $7.26 \%$ divorced or separated. $22.28 \%$ stated that they live alone, while $77.72 \%$ stated that they live with others, either with their spouses, or with their children, or in institutions for the elderly. It seems that the majority of patients lives with others and therefore has someone who can take care of them and support them. However, according to Damigo D, et al. the psychological support and care of the specialists is necessary for the improvement of the QOL of both the patients themselves and their families [10]. Regarding the professional status of the participants, the majority were retired (72.54\%) and out of work $(17.62 \%)$. It is obvious that dialysis limits the patient professionally and this finding agrees with Kaitelidou D, et al. who in their research concluded that dialysis is associated with high productivity loss [11]. Similar findings were recorded by Kastrouni M, et al. who in their research found that $43.6 \%$ of dialysis patients stopped working due to $\mathrm{CRF}$ [12].

There are many concomitant health problems that dialysis patients have that strain them as a result of which they have a negative effect on their QOL. In particular, hypertension, diabetes and heart problems plague them to a greater extent. $51.30 \%$ of patients suffered from hypertension, $32.12 \%$ with diabetes mellitus and $30.60 \%$ with heart problems. Based on what the participants reported, and the information contained in their medical records, the primary cause of CRF in the majority of the sample was hypertension and diabetes mellitus. Specifically for $21.24 \%$ of patients it was hypertension, for $17.62 \%$ hypertension and diabetes mellitus and for $13.99 \%$ diabetes mellitus. These are followed by medication, polycystic ovary disease and kidney stones. 
Table 4: Percentage comparison of vascular access complications in patients with AA and CVC (N=183).

\begin{tabular}{|c|c|c|c|c|c|}
\hline & $\begin{array}{l}\text { Patients with AA } \\
\qquad(\mathrm{N}=111)\end{array}$ & $\begin{array}{l}\text { Patients with CVC } \\
\qquad(\mathrm{N}=72)\end{array}$ & P-value & $\begin{array}{c}\text { Percentage } \\
\text { Difference (PD) }\end{array}$ & $\begin{array}{c}95 \% \\
\text { Confidence interval }\end{array}$ \\
\hline Mechanical Pneumothorax & $0(0 \%)$ & $0(0 \%)$ & - & - & - \\
\hline Artery subclavian puncture & $0(0 \%)$ & $1(1,39 \%)$ & - & - & - \\
\hline Venous subclavian rupture & $0(0 \%)$ & $0(0 \%)$ & - & - & - \\
\hline Venous subclavian stenosis & $0(0 \%)$ & $0(0 \%)$ & - & - & - \\
\hline Hemothorax & $0(0 \%)$ & $0(0 \%)$ & - & - & - \\
\hline Thrombosis & $0(0 \%)$ & $0(0 \%)$ & - & - & - \\
\hline Air embolism & $0(0 \%)$ & $1(1,39 \%)$ & - & - & - \\
\hline Poor catheter placement & $0(0 \%)$ & $0(0 \%)$ & - & - & - \\
\hline \multicolumn{6}{|l|}{ Infection } \\
\hline Local & $2(1,80 \%)$ & $21(29,17 \%)$ & $<0,001^{* *}$ & 27,37 & $(17,08,38,78)$ \\
\hline Bacteremia & $5(4,50 \%)$ & $32(44,44 \%)$ & $<0,001^{* *}$ & 39,94 & $(27,68,51,69)$ \\
\hline Osteomyelitis & $0(0 \%)$ & $0(0 \%)$ & - & - & - \\
\hline Endocarditis & $0(0 \%)$ & $0(0 \%)$ & - & - & - \\
\hline Thrombosis & $8(7,21 \%)$ & $2(2,78 \%)$ & 0,198 & 4,43 & $(-32,20,-11,11)$ \\
\hline \multicolumn{6}{|l|}{ Vascular access dysfunction } \\
\hline Low arterial supply & $4(3,60 \%)$ & $30(41,67 \%)$ & $<0,001^{* *}$ & 38,06 & $(26,14,49,80)$ \\
\hline Increased venous pressure & $0(0 \%)$ & $7(9,72 \%)$ & $0,001^{*}$ & 9,72 & $(3,76,18,74)$ \\
\hline Increased recirculation & $0(0 \%)$ & $0(0 \%)$ & - & - & - \\
\hline Insufficient clearance & $0(0,00 \%)$ & $1(1,40 \%)$ & - & - & - \\
\hline Narrowing/Obstruction of central veins & $8(7,21 \%)$ & $13(18,06 \%)$ & $0,024 *$ & 10,85 & $(1,24,21,85)$ \\
\hline Artery or nerve injury & $0(0 \%)$ & $0(0 \%)$ & - & - & - \\
\hline Hemorrhage & $3(2,70 \%)$ & $5(6,94 \%)$ & 0,170 & 4,24 & $(-2,08,12,73)$ \\
\hline Ischemic neuropathy & $0(0 \%)$ & $0(0 \%)$ & - & - & - \\
\hline Insufficient development of dilated veins & $5(4,50 \%)$ & $0(0 \%)$ & 0,248 & 3,12 & $(-3,27,8,84)$ \\
\hline Aneurysm formation & $27(24,32 \%)$ & $0(0 \%)$ & $<0,001^{* *}$ & 24,32 & $(15,66,33,08)$ \\
\hline Hand ischemia & $1(0,90 \%)$ & $0(0 \%)$ & - & - & - \\
\hline Subclavian syndrome & $3(2,70 \%)$ & $0(0 \%)$ & - & - & - \\
\hline Finger cramping & $1(0,90 \%)$ & $0(0 \%)$ & - & - & - \\
\hline Spinal infection & $0(0 \%)$ & $1(0,40 \%)$ & - & - & - \\
\hline Finger pain & $1(0,90 \%)$ & $0(0 \%)$ & - & - & - \\
\hline
\end{tabular}

The length of time patients with CRF are on a haemodialysis program plays an important role in their QOL [13]. The results of the survey showed that $31.09 \%$ of the participants were less than or equal to one year, while $67.36 \%$ more than one year. It is obvious that the majority follow a haemodialysis program for more than a year.

Regarding the type of vascular access of the sample, $57.51 \%$ of the participants had AA, 37.31\% CVC and 5.18\% AG. Therefore, the majority of patients had AA, but comparing the two provinces, it is noteworthy that in the province of Larnaca, CVC prevails in contrast to the province of Nicosia, where AA prevails. The results of the study showed that the QOL of haemodialysis patients is generally moderate. However, as far as the physical health factor is concerned, they presented low scores, which indicates that the most serious problems that dialysis patients face are related to the physical function of their body. In contrast to the environmental factor, they presented relatively high scores in relation to the other factors. In addition, the comparison between women and men showed that women have lower levels of QOL.

Comparing the averages of QOL factors in haemodialysis patients with AA and CVC in the sample, it was found that there is a statistically significant difference between the two types of vascular access to the mental health factor. It was found that the mental health of haemodialysis patients with CRF is in a worse state than the mental health of haemodialysis patients with AA. This shows that the problems that arise due to CRF more affect the mental state of the patient.

On the contrary, there was no statistically significant difference in the factors of physical health, social relations and the environment, as well as in the total score of QOL, as a result of which the comparison is not possible.

The results of the study showed that the percentage of patients who did not have complications in their vascular access was higher in patients with AA (48\%) than in patients with CVC (22\%). More specifically, in the percentage comparison of vascular access complications in patients with AA and CVC $(\mathrm{N}=183)$ it was found that infections in patients with CVC had the highest rates. Specifically, $44.44 \%$ of patients with CVC showed bacteremia compared to $4.50 \%$ with AA and $29.7 \%$ of patients with CVC presented local infection compared to $1.80 \%$ with AA.

Regarding the problems with the function of the vascular access, again the patients with CVC presented the most complications. Specifically, $41.67 \%$ of patients with CVC showed low arterial flow during dialysis compared to $3.60 \%$ of patients with AA and $9.72 \%$ increased venous pressure, while patients with AA did not experience 
this problem. In addition, $1.40 \%$ of patients with CVC had inadequate clearance. Central venous stenosis and obstruction occurred in $18.06 \%$ of patients with CVC compared to $7.21 \%$ of patients with AA. Aneurysms occurred only in patients with AA at a rate of $24.32 \%$ since patients with CVC do not have to deal with this problem.

During the attempt to place the CVC, two mechanical complications occurred, which consist of the puncture of the subclavian artery $(1.39 \%)$ and the air embolism (1.39\%). Spine infection was recorded in a patient with CVC $(1.40 \%)$ while no case was reported in a patient with AA. Bleeding episodes occurred in $6.70 \%$ of patients with CVC compared with $2.70 \%$ of patients with AA. Thrombosis occurred in $7.21 \%$ of patients with AA compared to $2.78 \%$ of patients with CVC. Patients with AA also had eavesdropping syndrome (2.70\%), finger cramps $(0.90 \%)$, finger pain $(0.90 \%)$ and ischemia of the hand $(0.90 \%)$. Patients with CVC are more likely to have complications with infections (local and bacteremia), vascular access problems (low blood pressure, increased venous pressure), and central venous stenosis and obstruction compared to patients with AA.

Almost all problems and complications of vascular access need to be addressed either during according to the results of this research, it is found that the problems and complications of vascular access in patients with CVC are more than the problems of patients with AA and contribute to the increase of hospital care and costs. Problems with the vascular access function of patients with CVC during dialysis are of increasing concern to nursing and medical staff in relation to the complications of patients with AA. Dialysis, at home by giving the patient treatment, or by admitting the patient to inpatient care. Home treatment is given to dialysis patients with CVC at a rate four times higher than to dialysis patients with AA. Also worrying are the rates of hospital admissions of patients with CVC compared to the admissions of patients with AA.

In the dialysis unit of the General Hospital of Larnaca, Povidone Iodine $10 \%$ and Alcohol $70 \%$ are used for antiseptic entry of the vascular access, while in the unit of the General Hospital of Nicosia, octenisept ${ }^{\circ}$ is used. Chlorhexidine solution according to the international literature has much better results and its use is recommended [14,15], but unfortunately it is not used by any province. Mimoz $\mathrm{O}$, et al. support the use of Chlorhexidine solution for the care of CVC after their research showed that it reduced the rates of bacteremia and colonization of CVC by about 50\% compared to the Povidone Iodine solution [16].

\section{Conflict of Interest}

None.

\section{References}

1. Nettleton S (2006) The Sociology of Health and Illness. Wiley, United States.

2. Sathvik BS, Parthasarathi G, Narahari MG, Gurudev KC (2008) An assessment of the quality of life in hemodialysis patients using the WHOQOL-BREF questionnaire. Indian J Nephrol 18: 141-149.

3. Georgiades G, Kandarzi K, Vargemezis V, Lazaridi M (2007) The history of vascular access in hemodialysis patients. From the early days of Willem JK off to the present. Arch Hell Med 24: 389-397.
4. The Kidney Foundation of Canada (2015) Canadian Organ Replacement Register Annual Report: Treatment of End-Stage Organ Failure in Canada, 2004 to 2013.

5. Ginieri-Coccossis M, Triantafillou E, Tomaras V, Soldatos C, Mavreas V, et al. (2012) Psychometric properties of WHOQOL-BREF in clinical and healthy Greek populations: incorporating new culture-relevant items. Psychiatriki 23: 130-142.

6. Castledinea C, Casulaa A, Fogar D (2012) UK Renal Registry $14^{\text {th }}$ Annual Report: Chapter 2 UK RRT Prevalence in 2010: National and Centre-Specific Analyses. Nephron Clin Pract 120: c29-c54.

7. Panagopoulou A (2009) Lifestyle characteristics of patients with chronic renal failure undergoing renal function replacement therapy with haemodialysis, peritoneal dialysis or undergoing successful renal transplantation.

8. Pelayo RA, Cobo JLS, Reyero ML, Sáenz ABP, Tovar AR, et al. (2011) Repercusión del acceso vascular sobre la calidad de vida de los pacientes en tratamiento con hemodiálisis. Rev Soc Esp Enferm Nefrol 14: 242-249.

9. Grilla E (2012) Assessment of quality of life in patients with chronic disease. The case of patients with end-stage chronic renal failure undergoing dialysis.

10. Damigos D, Kaltsouda A, Ikonomou M, Siamopoulos KC (2010) Biopsychosocial approach to chronic kidney disease. The role of the Psychonephrology Unit. Hell Society Nephrol 22: 120-129.

11. Kaitelidou $\mathrm{D}$, Liaropoulos $\mathrm{L}$, Siskou $\mathrm{O}$, Mamas $\mathrm{T}$, Zirogiannis $\mathrm{P}$, et al. (2007) The social and economic consequences of dialysis in patients' lives with chronic renal insufficiency. Nursing 46: 246-255.

12. Kastrouni M, Sarantopoulou E (2008) Study of quality of life of patients who suffer from chronic renal failure.

13. Anees M, Hameed F, Mumtaz A, Ibrahim M, Khan MNS (2011) Dialysis-related factors affecting quality of life in patients on hemodialysis. Iran J Kidney Dis 5: 9-14.

14. Centers for Disease Control and Prevention (2014) Top CDC Recommendations to Prevent Healthcare-Associated Infections. United States.

15. Centers for Disease Control and Prevention (2006) Central Venous Catheter Hub Cleaning Prior to Accessing. United States.

16. Mimoz O, Villeminey S, Ragot S, Dahyot-Fizelier C, Laksiri L, et al. (2007) Chlorhexidine-Based Antiseptic Solution vs Alcohol-Based Povidone-lodine for Central Venous Catheter Care. Arch Intern Med 167: 2066-2072. 